\title{
Reversible, Unilateral Dilatation of Upper Urinary Tract due to a Tight Waist String
}

\author{
E. T. Kavin Kardhikh • Chandrasekaran Venkatesh • \\ Nanda Chhavi • Dhandapany Gunasekaran • \\ Palanisamy Soundararajan
}

Received: 17 September 2011 /Accepted: 25 November 2011 /Published online: 10 December 2011

(C) Dr. K C Chaudhuri Foundation 2011

Sir,

A 5-mo-old male infant was brought with history of cough, cold and fever of $2 \mathrm{~d}$ duration and excessive cry for $3 \mathrm{~h}$ prior to admission. There were no similar complaints in the past. On examination, the baby was febrile, fussy and crying excessively. His vitals were normal. Examination was unremarkable except for a palpable liver $2 \mathrm{~cm}$ below right costal margin and a distended urinary bladder due to a tight waist string. On removing the waist string, the baby passed large quantity of urine and stopped crying immediately. Blood investigations were all normal except for a positive C-reactive protein $(2.4 \mathrm{mg} / \mathrm{dl})$. The blood and urine cultures were sterile. Ultrasound (USG) abdomen showed mild hydronephrosis on the left side. The infant received injection ceftriaxone for $7 \mathrm{~d}$ and discharged on chemoprophylaxis with co-trimoxazole. At follow up one mo later, repeat USG and micturiting cystourethrogram (MCUG) were done and both were normal. Hence chemoprophylaxis was stopped and a diagnosis of transient unilateral hydronephrosis secondary to bladder outlet obstruction was considered.

Constriction to bladder outlet by a waist string causing unilateral upper urinary tract dilatation has not been reported in literature to the best of our knowledge. Unilateral VUR has been described in literature in upto $35 \%$ boys with PUV (Posterior Urethral Valves) and is associated with protected renal function [1]. In our case PUV was ruled out by the presence of a normal MCUG and absence of bladder

E. T. K. Kardhikh · C. Venkatesh · N. Chhavi • D. Gunasekaran •

P. Soundararajan

Department of Pediatrics,

Mahatma Gandhi Medical College and Research Institiute,

Pillaiyarkuppam,

Puducherry, India

C. Venkatesh $(\square)$

Plot No:5, Second Street, Jhansi Nagar,

Puducherry 605004, India

e-mail: cvenkatesh@hotmail.com trabeculation and hypertrophy which are typically seen in a valve bladder [2].Transient VUR due to retention of urine could be missed by a delayed MCUG and hence its possibility cannot be ruled out completely. Intermittent ureteropelvic junction obstruction can also present as transient, recurrent hydronephrosis with a characteristic sonographic finding of a thickened pelvic wall seen during recovery [3]. The diagnosis can be missed during a pain free episode. However in our case, USG did not show thickened renal pelvis. Unilateral hydronephrosis detected by ultrasonography on two patients with lower urinary tract obstruction has also been described in literature [4] associated with renal impairment unlike in our case where the hydronephrosis was only transient and did not result in renal impairment. An obstructed megaureter can mimic hydronephrosis but has a short segment which is aperistaltic with a normal juxta vesical junction and normal insertion [5]. However the dilatation is persistent unless treated surgically.

Tight waist strings can result in bladder obstruction and retention of urine. A transient VUR is likely to be missed by a delayed MCUG. A high index of suspicion and knowledge of traditional practices that are prevalent in the society are useful skills in management of cases such as these.

\section{References}

1. Donnelly LF, Gylys-Morin VM, Wacksman J, Gelfand MJ. Unilateral vesicoureteral reflux: association with protected renal function in patients with posterior urethral valves. AJR Am J Roentgenol. 1997;168:823-6.

2. Chatterjee SK, Banerjee S, Basak D, et al. Posterior urethral valves: the scenario in a developing center. Pediatr Surg Int. 2001;17:2-7.

3. Tsai JD, Huang FY, Lin CC, et al. Intermittent hydronephrosis secondary to ureteropelvic junction obstruction: clinical and imaging features. Pediatrics. 2006;117:139-46.

4. Abott KC, Wortham WC. Ultrasonographic unilateral hydronephrosis with bladder outlet obstruction. Clin Nephrol. 1994;42:337-40.

5. Meyer JS, Lebowitz RL. Primary megaureter in infants and children: a review. Urol Radiol. 1992;14:296-305. 